\title{
On the Status of Social Support System for Non-Local Families with Leukemia Children
}

\author{
Max Q.-Man Wang, Yan Ming \\ Department of Sociology and Social Work, Capital Normal University, Haidian, Beijing, China \\ wm1204@sina.cn, mingyan@cnu.edu.com
}

\begin{abstract}
Leukemia is a costly serious illness. As for the families with Leukemia children, social support system plays a vital role to get successful cure. The author interviews 8 non-local families that are receiving treatment in a hospital in Beijing and uses qualitative analysis to study their social support system. The main conclusions are: 1 . The structure of micro-supporting system is relatively complete, however, the support is kind of limited. When the curing process goes on, the support weakens. 2. The macro-support system is mainly economic support with consistency. However, the supporting power needs further improvement.
\end{abstract}

Keywords - families of children with leukemia, social support system

\section{外地来京白血病患儿家庭的社会支持系统 及其特征研究}

\author{
王曼明艳 \\ 首都师范大学政法学院社会学与社会工作系, 海淀, 北京, 中国
}

\begin{abstract}
摘 要 白血病是一种治疗周期长且费用高的严重疾病, 患儿家庭社会支持系统的构建状况对其能否顺利治疗起着至关重要的作 用。笔者运用观察法和访谈法在北京某医院对非京籍白血病患儿家庭开展调查, 运用定性方法对患儿家庭社会支持系统现状、特点和 所存在的问题进行研究。主要结论是: 1. 患儿家庭微观支持系统的结构比较健全, 但整体支持力度有限, 且随着治疗周期的延续, 呈 现逐渐减弱的趋势。2. 患儿家庭支持的宏观系统以经济支持为主, 持续性较强, 但支持力度有待提高。
\end{abstract}

关键词 白血病患儿家庭，社会支持系统

\section{1. 研究背景与意义}

白血病是一种恶性程度极高的血液病, 目前儿童发病 率为 4-6/10 万人. 通过化疗、造血干细胞移植等方式, 80\%-90\% 可以缓解, 60\%-70\%可以治愈。白血病的治疗有三 大特点: 1) 费用高, 平均需要 10-30 万, 骨髓移植需要 30-100 万。2) 周期长, 一般需要 2-3 年时间。3) 对医疗水 平要求高, 很多外地的地方医院都无法提供标准的医疗环 境, 极易导致患儿感染。

在治疗过程中, 患儿家庭承受压力只依靠家庭内部的 力量是不可能缓解的, 所以社会支持系统就至关重要。社 会支持系统会影响患儿家庭对治疗方案的选择, 甚至决定 患儿是否可以长期接受治疗。健全的社会支持系统有助于 患儿家庭获得更多资源, 更好地应对在治疗的过程中遇到 的困难, 把疾病带给白血病患儿及其家庭的危害降到最低。
因此，患儿家庭社会支持系统的现状和特征非常值得关注。

\section{2. 文献回顾}

本研究以林南等学者提出的社会支持理论为依据, 即 “意识到的或实际的由社区、社会网络和亲密伙伴提供的 工具性或表达性资源。”在这一定义中可以区分社会支持的 宏观、中观、微观层面, 以及主观和客观层面。

关于白血病患者研究的分析工具, 多采用 “社会支持 评定量表 (SSRS)、领悟社会支持量表 (PSSS)、家庭 APGAR 问卷” 等工具。但这些量表中的问题比较固定, 并非针对 白血病患者量身设定, 有些问题套用在白血病患儿家庭这 个特定的研究群体上, 不是十分合适。

以往白血病患儿家庭社会支持系统研究的结论有: 1 . 多数家庭主要通过亲属和朋辈等微观支持系统寻求关心和 
帮助，而社会组织、政府及其他的社会支持较少。2. 从支 持的强度来看, 体制性系统支持力量的有限性, 使白血病 患儿家庭的社会支持系统表现出 “内倾化” 的特点。 ${ }^{[11}$

总体上看看，1）针对白血病患儿家庭社会支持相关的 医学、心理学等研究的深度和针对性都有待进一步加强。2) 较少从慈善团体、社会工作者和政府政策等社会综合环境 来考察个体或群体的社会支持系统形成的特点及存在的问 题。3) 以往研究的重点往往放在社会支持系统的模式、结 构或功能等要素上, 较少关注被研究对象的内心深层体验。 由此, 笔者针对外地来京白血病患儿家庭的真实情况设计 一套访谈提纲, 通过定性分析, 比较深入地探讨其社会支 持系统的特点和问题。

\section{3. 研究设计}

本研究以在北京某医院接受治疗的白血病患儿家庭为 研究对象, 随机抽取了 8 个非京籍的患儿家庭进行访谈, 患 儿的平均年龄在 8 岁左右。

笔者将患儿家庭的社会支持系统分为微观社会支持系 统和宏观社会支持系统两大部分。微观社会系统支持主要 是指白血病患儿家庭内部、朋辈和志愿者等来自个体的相 对微观的系统提供的支持。也即没有制度性的规定, 主要 是靠情感和血缘来维持的支持。包括亲属支持、朋辈支持、 病友支持、社会工作者支持几方面, 内容涉及经济支持、 心理支持、信息咨询等。本文将分析这几种支持在内容和 力度上的差异。宏观系统社会支持主要指除了亲友以外, 来自组织、制度、政策等方面的支持, 包括政府福利保障、 单位、社会公益救助组织、社会媒体等方面的支持, 内容 涉及经济支持、信息传递等。

\section{4. 外地来京白血病患儿家庭社会支持系统现状}

4.1 微观社会支持系统现状

\subsection{1 亲属支持}

亲属为外地来京白血病患儿家庭提供的支持主要为经 济资助和日常家人照养这两方面, 其中经济支持比较显著。

经济支持方面, 8 个家庭中有 6 个都得到了亲属一定 的经济支持, 另 2 个由于出嫁、外出打工等原因与亲属联 系较弱, 没有获得亲属支持。但随着治疗周期的延续, 患 儿家庭得到亲属经济上的支持明显减弱。

日常家人照养方面, 患儿父母的兄弟姐妹往往会承担 起照顾患儿祖辈和患儿家庭其他子女的责任。笔者调查的 8 个家庭中有 6 个是多子女家庭, 其他子女均处于学龄阶 段, 需要成年人的照养。这时患儿的亲属往往承担这一任 务, 帮助解除患儿父母的后顾之忧。

心理支持方面, 患儿家庭从亲属处得到的情感支持较
弱。原因是 1）空间距离阻隔了亲人面对面的沟通。2）亲 属很难前往医院看望或照料患儿。3）跟亲属倾诉内心的苦 楚只会徒增他们的担心。4）在最初得知患儿患病时每个亲 属都会给予一定安慰和鼓励, 但随着治疗周期的延长和病 情的反复, 亲属对患儿家庭的心理支持逐渐减弱。

\subsection{2 朋辈支持}

患儿家庭的朋辈支持在各方面都呈现较弱的状态。

与亲属相比, 患儿家庭接受到的来自朋友的经济支持 较少。被访家庭中有 2 个表示曾向关系比较亲密的朋友借 钱, 但是金额不大。他们觉得无论经济债或人情债, 自己 将来要是还不上就不愿去借。这与家庭本位的文化传统有 很大关系。社会团结首先是家庭 (家族) 的团结, 是靠差 序格局形成的社会支持体系。'21

朋友的心理支持也较弱。只有一位家庭的母亲表示自 己会向好朋友倾诉不良情绪, 其他的患儿父母均表示不会 和朋友诉说。由于患儿不在老家治病, 朋友也很少打电话 询问情况, 患儿父母认为偶尔的联系并不能缓解他们内心 的压力, 所以也无意诉说。

\subsection{3 病友支持}

病友之间的相互交流成为患儿家庭主要的心理支持, 这种交流过程也是经验和信息的传递。对白血病患儿父母 来说, 最佳的情感交流对象是病友。病友之间的关系因为 同病相怜和感同身受而变得亲密。笔者在医院长期观察发 现, 患儿刚刚确诊并入院治疗时, 是家长情绪最不稳定的 时期, 这时其他病友的安慰、开导和自身经验的分享对患 儿父母非常有用。治疗进入正轨后, 患儿家庭与病友长期 共处一室, 通过聊天加深了解, 在生活上互帮互助, 时间 久后大家的情感日趋深厚, 愿意向对方倾诉自己的焦虑或 困惑。甚至出院期间也会打电话互相问候, 给予心理支持。

病友之间还有信息支持。比如护理患儿的经验, 共享 救助资源等, 还包括在医院附近生活购物、租房子的信息。

\subsection{4 医疗社会工作者支持}

专业的医疗社会工作者可以帮助患儿家庭调动各类资 源。经济方面, 医疗社工会帮助患儿家庭申请各项社会救 助, 指导家长准备申请材料等, 还在大学校园里为经济极 度困难的患儿开展募捐活动。

医疗社工也关注患儿及其父母的心理和日常生活状 况。他们会陪伴孩子, 疏导患儿与父母的不良情绪。或者 定期举办一些文娱活动, 缓解患儿家庭的心理压力。医疗 社工还调用各种资源丰富患儿及家长的生活, 帮助需要休 学进行治疗和复查的白血病患儿补习功课。

但目前专业医疗社会工作者只在某些医院才有, 并不 是白血病患儿家庭普遍拥有的社会支持。同时, 医疗社工 
的服务目前处于初步探索阶段, 其本身可以调动的时间、 精力、资源有限, 专业性有待进一步的提高。

\section{2 宏观社会支持系统现状}

\subsection{1 政府社会保障体系提供的支持}

理论上, 患儿目前可以享有的医疗保障主要有新型农 村合作医疗、城乡医疗救助、重大疾病医疗保障、父母单 位家属半劳保、合作医疗。基本医疗保险（即新型农村合 作医疗和城乡医疗救助, 以下将两者统称为 “基本医疗保 险”）目前的覆盖率约为 95\% 左右，但基本医疗保险报销 的比例低, 且可报销的最高限额也较低, 白血病的报销比 例在 60\%-70\%, 跨省报销的比例在 30\%左右。

患儿家长对基本医疗保险的政策了解较多, 有 7 位家 长表示正在使用新农合, 一位家长表示在使用城乡医疗救 助。八位家长均表示没有听说过重大疾病医疗保障, 可能 是因为该政策的覆盖率还不是很高。

政府的医疗保障制度对白血病患儿家庭在经济方面起 到了一定的支持作用, 但仍存在的一些问题: 1) 跨省报销 的比例较低, 两种基本医疗保险跨省报销比例都会缩减 30\% 左右。2) 报销有诸多限制, 比如有些在报销范围之内的药, 如果医院不进, 患儿家庭自己购买的话也要自费处理。挂 号费、针管等医疗器械费用、病床费等都不在报销范围之 列。3）报销手续较为繁琐, 要回到户籍所在地去办。4） 报销的周期较长, 笔者采访的患儿家庭有的在进行第二个 疗程的收尾治疗, 但是还没有拿到过任何报销所得的钱。

\subsection{2 单位提供的支持}

单位支持主要是指来自患儿父母单位和患儿学校的支 持, 二者可以通过内部募集捐款的形式提供。但农村患儿 家庭接受单位帮助的机会较小, 即使有, 支持力度也较弱。 在被调查的 8 个家庭中, 只有 2 个家庭得到过单位的经济 支持。其中一个患儿父亲的单位发起捐款活动, 筹集捐款 两万元左右。另一个患儿所在学校募捐了六万元左右。但 多数患儿学校和父母单位的救助较少。原因也在于患儿父 母大多从事个体经营或务农, 很少在固定的单位或公司工 作, 因此接受单位经济支持的可能性小。

\subsection{3 社会公益救助组织提供的支持}

在笔者开展调查的医院, 患儿可以申请的慈善组织救 助项目有：中国红十字基金会 “小天使基金” 的贫困白血 病儿童救助项目、新阳光慈善基金会的 “0-18 岁青少年 白血病救助行动”、神华公益基金会的 “神华爱心行动”。

以神华公益基金为例, 救助项目的特征有：1）救助对 象有限制, 一般为低危或中危病患。2) 救助款项仅用于患 儿的医疗费用, 救助款在医院结算, 不直接支付给个人或
家庭。救助款不是一次到位, 而是分期支付。

表 1 笔者访谈的八个患儿家庭接受的资助情况

\begin{tabular}{|c|c|}
\hline & 受资助情况 \\
\hline 1 & 无 (处于第一个疗程, 正在准备申请材料) \\
\hline 2 & 无 (属于高危病患, 不符合申请条件) \\
\hline 3 & 2012 年申请到 “小天使” 基金 4 千元救助款 \\
\hline 4 & 无（正在递交救助申请, 等待审批） \\
\hline 5 & $\begin{array}{c}2012 \text { 年申请到神华基金 } 5 \text { 万元救助款, 现已到账 } 2.5 \text { 万。 } \\
\text { 同时以递交 “小天使” 基金的申请材料, 等待审批。 }\end{array}$ \\
\hline 6 & 2012 年申请神华基金 6.5 万救助款, 现已到账 3.25 万 \\
\hline 7 & 无（属于高危病患, 不符合申请条件) \\
\hline 8 & 2012 年申请神华基金 5 万元救助款 \\
\hline
\end{tabular}

而项目救助过程则表现出这样一些问题: 1) 从申请到 获批再到资金到位的时间过长, 尤其是资金到位的时间比 较长。2) 救助项目的知晓度有待提高, 除了神华基金与医 院直接联系, 使得患儿家庭申请救助的渠道畅通以外, 其 他的救助项目患儿家长很少知道。这是很有代表性的问题, 很多患重大疾病的家庭没有申请社会救助主要是因为根本 不知道有此种救助更不知道申请救助的具体方法与途径。

3) 患儿家长申请救助的模式一般为: 患病和治疗初期, 家 长沉浸在痛苦和懵懂之中, 缺乏申请救助的意识, 只会想 到在家庭内部寻求支持。随着治疗的深入, 家庭内部的支 持已经耗尽, 家长才会把注意力转向救助项目的支持。4) 项目资金不足。随着救助项目逐渐深入, 需要救助的家庭 增多，有些基金会的救助项目已因资金匮乏而终止。

\subsection{4 媒体提供的支持}

媒体作为信息发布的平台可以为白血病患儿家庭带来 一定的间接支持, 即媒体并不直接给予患儿家庭经济支持, 而是通过信息平台, 发布患儿需要救助的信息, 帮助患儿 得到社会各界爱心人士的支持。但媒体提供的这种支持带 有偶然性, 得到媒体帮助机会的比例较小, 要想争取到媒 体的资源有一定困难。

\section{5. 外地来京白血病患儿家庭社会支持系统的特点}

5.1 微观支持系统的特点

1）患儿家庭微观支持系统的结构比较健全, 其亲属、 朋辈、病友和社会工作者都不同程度上给予患儿家庭以支 持。患儿的亲属在日常家人照养上扮演者非常重要的角色。 病友和社工是患儿家庭最佳的情感交流对象, 他们除了给 予心灵上的支持，还会给予信息资源的支持。 
2) 患儿家庭微观系统整体支持力度有限, 且来源比较 单一和固定。经济支持主要支持来自患儿家庭的亲属。亲 属的经济支持虽然具有无需偿还的特点, 但是外地来京白 血病患儿的家庭大多来自农村, 其亲属自身的经济实力较 弱，所以资助的金额较小。朋辈的经济支持力度更为薄弱。 心理支持方面，虽然微观支持的各个主体都有给予，但是 只有病友和志愿者对患儿家庭的心理支持起到了较强的作 用, 患儿家庭的心理支持来源比较固定和单一。

3) 随着治疗周期的延续, 微观系统的支持呈现出逐渐 减弱的趋势。微观支持体统主要来自个体, 其可调动的资 源有限，随着治疗周期的延长，其可以提供的支持也在不 断削弱。所以从长远的角度来看, 单纯依靠微观社会系统 的支持对患儿家庭来说是远远不够的, 还需要宏观的社会 支持系统给予可持续的支持。

\section{2 宏观支持系统的特点}

1) 患儿家庭支持的宏观系统以经济支持为主, 支持的 可持续性比较强。例如医疗保障政策和社会公益救助组织 的支持力度，不会随着患儿治疗周期的持续而削减。

2) 部分宏观支持系统的获得较为困难。例如来自父母 单位的支持较少; 医保报销资金到帐周期较长, 社会公益 救助组织的资金获批后到位缓慢; 虽然社会公益救助组织 的救助项目较多, 但患儿家庭知道的有限; 有些项目申请 的条件较为严格, 比如不向被诊断为高危的患儿提供救助; 另外媒体作为信息平台提供的间接支持具有偶然性，患儿 家庭缺乏向媒体求助的意识, 而且要想争取到媒体的资源 有一定困难。

3) 宏观支持系统的支持力度有待提高。政府社会保障 方面的支持跨省治疗报销的比例会降低 30\%左右，很多治 疗开销都不在报销范围之内。而且随着公益基金会开展救 助活动时间的延长，一些基金会的资金都处于溃乏状态， 对患儿家庭的支持力度减弱。

\section{6. 讨论}

\section{1 外地来京的农村家庭更需要社会的大力支持}

医疗费用问题是困扰白血病患儿家庭的重要问题。部 分患儿家庭来自农村, 以务农为主业，其自身的经济收入 微薄且不稳定，其亲属和朋辈大多与其情况相似，可以提 供的支持十分有限。很多农村家庭不堪重负, 最终放弃治 疗。所以他们更加需要引起社会的关注。例如可以由专业 医疗社会工作者帮助其创造新的社会支持，使其更能顺利 的持续治疗。

\section{2 政府在医疗保障政策方面需要进一步改善}

在医疗保障政策方面，首先，应当加快惠民医疗保险 制度的推行, 并即时对政策做出调整, 使其真正惠及到有 迫切需求的群体。其次，应适当提高农村患儿医药费用的 报销比例, 并扩大报销药品种类的范围, 使患儿家庭的医 疗费用在其大多数家庭可以承受的范围之内。再次, 加强 医药市场的规范化管理, 适当调低白血病患儿治疗用药的 价格。

\section{3 加强医疗社会工作者专业队伍的建设}

目前医疗社会工作的普及率有待提高。部分引医疗社 工的医院, 医疗社工也多是由大学中的老师和学生组成, 以志愿者或兼职社工的身份进入医院。其可调动的资源有 限、工作缺乏连续性等问题, 使其专业性和认可度受到影 响。

应该在医院内部设立专门的社工岗位, 由专业全职社 工为患儿和医院提供更加稳定和专业的服务。其次, 在设 立全职社工岗位的基础上，可以引进一些兼职人员或志愿 者，这样可以调动更多的资源服务于患儿家庭。但是为了 确保其服务的专业性，需要对其进行一些必要的培训，使 其了解专业的医疗社会工作的内容。

\section{参考文献}

[1] The China Youth University for Political Science Youth Research Institute, “A Report On The Living Conditions Of Children Diagnosed With Leukemia In Rural China," Chinese red cross foundation, 2013.

[2] The China Youth University for Political Science Youth Research Institute, “A Report On The Participation Of Charitable Organizations In Medical Aid For Needy Children In China," Chinese red cross foundation,2013.

[3] Jing Zhang, "A Research into the Social Support System to Family with Leukemia Child," WanFangdata, 2006.

[4] M. Young, The Technical Writer's Handbook. Mill Valley, CA: University Science, 1989.

[5] Yingfeng Zhou and Ying Wang, "Perceived Social Support of Hospitalized Children with Leukemia," Journal of Nursing Science, Vol.18, No.10 Oct. 2003

注释:

【1】 Jing Zhang, "A Research into the Social Support System to Family with Leukemia Child," WanFangdata, 2006.

【2】 Shuming Liang, "The essence of Chinese culture," Academic Press, 1987. 